\title{
Comparison of SSI with APCI as an Interface of HPLC-Mass Spectrometry for Analysis of a Drug and its Metabolites
}

\author{
Tetsuya Arinobu, Hideki Hattori, Hiroshi Seno \\ Department of Legal Medicine, Aichi Medical University School of Medicine, Aichi, Japan
}

\author{
Akira Ishii \\ Department of Legal Medicine and Bioethics, Nagoya University Graduate School of Medicine, Nagoya, Japan \\ Osamu Suzuki \\ Department of Legal Medicine, Hamamatsu University School of Medicine, Hamamatsu, Japan
}

\begin{abstract}
Sonic spray ionization (SSI) was compared with atmospheric pressure chemical ionization (APCI) as an interface of high-performance liquid chromatography (HPLC)-mass spectrometry (MS) for sensitive analyses of a neuroleptic drug, haloperidol and its two metabolites, such as reduced haloperidol and 4-(4-chlorophenyl)-4-hydroxypiperidine (CPHP), in biological samples. For both SSI and APCI interfaces, HPLC-MS-MS gave higher sensitivity than HPLC-MS. The sensitivities by HPLC-SSI-MS-MS for haloperidol and reduced haloperidol were 100 and 30 times higher, respectively, than those by HPLC-APCI-MS-MS; no spectrum with recognizable peaks was obtained for CPHP with the APCI interface. Therefore, detection limits and regression equations were examined by the HPLC-SSI-MS-MS for human plasma and urine samples spiked with the above drug and its metabolites. Haloperidol, reduced haloperidol, and CPHP showed good linearity in the ranges of 5-800, 10-800, and 100-800 ng/mL, respectively, for both human plasma and urine; their detection limits were 2.5, 5, and 75 $\mathrm{ng} / \mathrm{mL}$, respectively, using a new polymer HPLC column which enabled direct application of biological samples. (J Am Soc Mass Spectrom 2001, 13, 204-208) (c 2001 American Society for Mass Spectrometry
\end{abstract}

S onic spray ionization (SSI) was developed in 1994 as a new technique to generate molecular ions under a very wide range of solvent systems and liquid flow rates [1, 2], and has become commercially available very recently as an interface of high-performance liquid chromatography (HPLC) and mass spectrometry (MS). The mobile phase is electrically neutral; however, in a small region, especially around the surface layer of the solution, charge separation can occur. In SSI, vaporization is done so that the surface layer of the solution, in the region of charge separation, is stripped by fast gas flow and electrically charged airborne droplets are created. The diameters of these electrically charged droplets shrink by vaporization of solvent molecules from the surface, and protonated molecular ions are formed in the gas phase. These ions enter the 3-dimensional quadrupole through the aper-

Published online January 15, 2002

Address reprint requests to Dr. T. Arinobu, Department of Legal Medicine, Aichi Medical University School of Medicine, Nagakute-cho, Aichi 4801195, Japan. E-mail: arinobu1@hotmail.com ture-1 and aperture-2 electrodes for mass analysis. Organic compounds are ionized by nebulizing sample solutions using a high-speed gas flow only, at room temperature [1, 3].

In this study, HPLC-SSI-MS(-MS) has been compared with HPLC-MS(-MS) with atmospheric pressure chemical ionization (APCI), for analysis of haloperidol and its two metabolites, reduced haloperidol and 4-(4chlorophenyl)-4-hydroxypiperidine (CPHP), in biological matrices to assess the utility of SSI in actual biomedical assays.

\section{Experimental}

\section{Materials}

Haloperidol, reduced haloperidol, and CPHP were commercially obtained from Research Biochemical International (Natick, MA). A haloperidol chlorinated analog, 4-[4-(4-chlorophenyl)-4-hydroxy-1-piperidinyl](4-chlorophenyl)-1-butanone, used as internal standard (I.S.) was also obtained from Research Biochemical 


\section{(A) SSI}
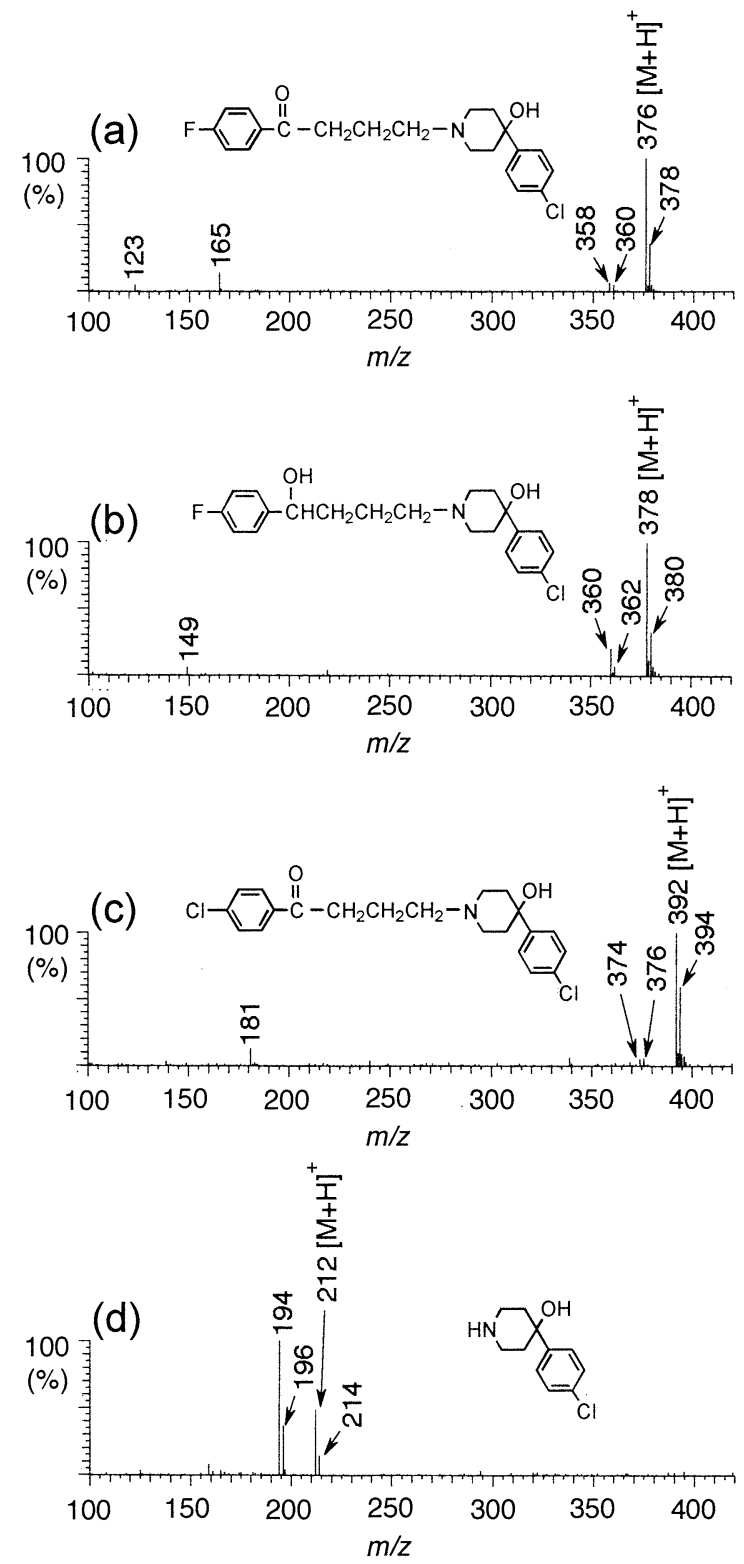

(B) $\mathrm{APCl}$
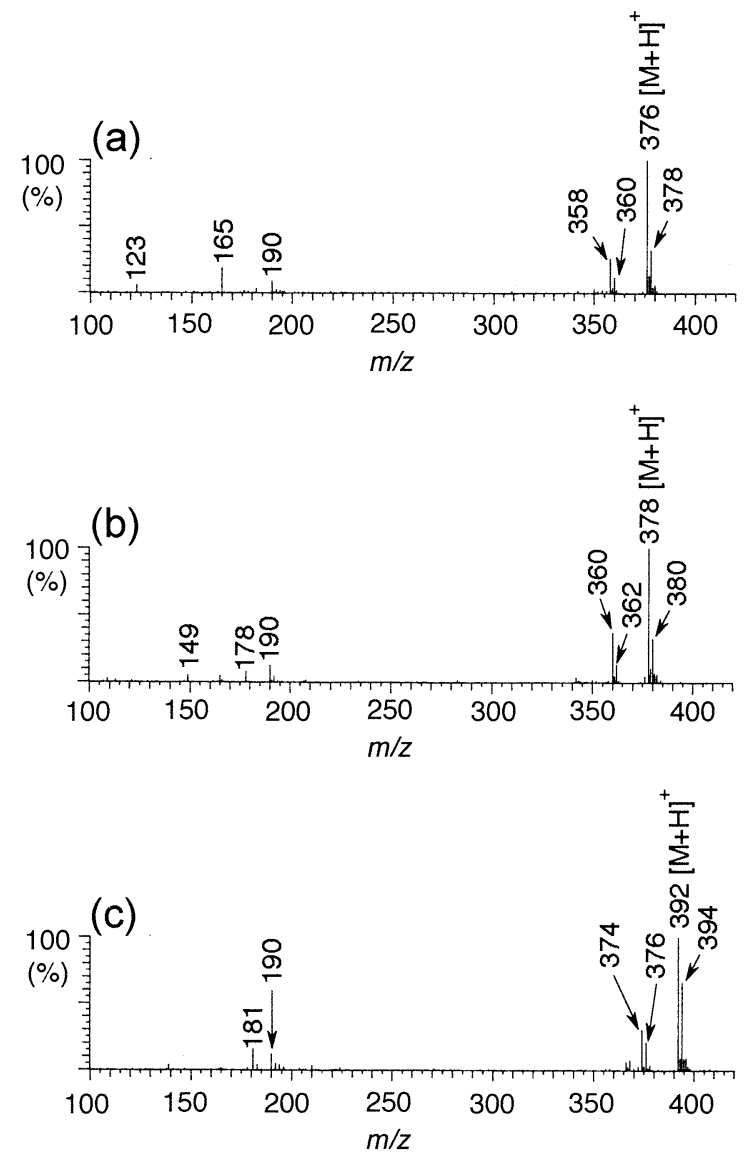

(d)

Figure 1. Comparison of mass spectra of haloperidol (a), reduced haloperidol (b), haloperidol chlorinated analog (c), and CPHP (d) obtained by SSI-HPLC-MS (A) with those by HPLC-APCI-MS (B). A spectrum with a recognizable peak was not obtained for CPHP with APCI, even with the injection of $62.5 \mu \mathrm{g}$ on-column.

International (Natick, MA). Reserpine was obtained from Sigma (St. Louis, MO). Other common chemicals used were of the highest purity commercially available. Whole blood and urine were obtained from healthy subjects. Whole blood was centrifuged at $1630 \mathrm{~g}$ for 15 min in the presence of EDTA-2Na (edetate disodium) to obtain plasma.

\section{MS-MS Conditions}

An M-8000 ion trap mass spectrometer (LC/3DQMS, Hitachi, Tokyo, Japan), equipped with SSI or APCI interface was used for analyses. The mass calibration was performed by flow injection of reserpine and haloperidol as mass makers. All spectrometric detection was made in the positive ionization mode. The MS-MS conditions for SSI interface were optimized by sequential flow injection of $1 \mathrm{ng} / \mu \mathrm{L}$ (in the initial HPLC mobile phase described below) of haloperidol, reduced haloperidol, the haloperidol chlorinated analog, and CPHP at a flow rate of 20 $\mu \mathrm{L} / \mathrm{min}$ using a syringe pump; the conditions for APCI interface were determined by sequential flow injection of $12.5 \mathrm{ng} / \mu \mathrm{L}$ (in the initial HPLC mobile phase) of each compound at a flow rate of $333 \mu \mathrm{L} / \mathrm{min}$. 
Table 1. CID product ion mass spectra of haloperidol, reduced haloperidol, haloperidol chlorinated analog, and CPHP obtained by $\mathrm{SSI}$ and $\mathrm{APCI}^{\mathrm{a}}$

\begin{tabular}{lll}
\hline & \multicolumn{1}{c}{ SSI-MS-MS } & \multicolumn{1}{c}{ APCI-MS-MS } \\
\cline { 2 - 3 } Compounds & \multicolumn{1}{c}{$\begin{array}{c}\text { CID product ion spectra } m / z \\
(\% \text { intensity) }\end{array}$} & \multicolumn{1}{c}{$\begin{array}{c}\text { CID product ion spectra } m / z \\
(\% \text { intensity) }\end{array}$} \\
\hline \hline Haloperidol & $165(100), 166(5)$ & $165(100), 166(5), 194(6)$ \\
Reduced haloperidol & $360(100), 361(12), 362(38), 363(6)$ & $360(100), 361(9), 362(40), 363(7)$ \\
Haloperidol chlorinated analog & $139(19), 181(100), 182(6), 183(22)$ & $139(15), 141(9), 181(100), 182(7), 183(33)$ \\
CPHP & $194(100), 196(31)$ & Not available \\
\hline
\end{tabular}

${ }^{a} \mathrm{CID}$ conditions are described in the text.

The MS-MS conditions optimized for haloperidol, reduced haloperidol, haloperidol chlorinated analog, and CPHP, for both SSI and APCI interfaces, were as follows, respectively. Step of ion isolation (MS 1): Isolation masses, $m / z$ 375.39-376.64, 377.38-378.65, 391.36-392.67, and 211.51-212.51; low mass cutoff, $\mathrm{m} / \mathrm{z}$ $368.49,370.45,384.17$, and 207.26; isolation time, 10.00 $\mathrm{ms}$ for all compounds; isolation voltage, 0.050, 0.050, 0.050 , and $0.015 \mathrm{~V}$. Step of collisionally induced dissociation (CID; MS 2): CID resonance, $m / z$ 368.09-384.26; 390.00-410.00, 383.38-401.02, and 210.50-244.00; low mass cutoff, $m / z$ 130.00, 150.00, 120.00, and 105.17; CID time, $50.00 \mathrm{~ms}$ for all compounds; CID voltage, 0.235 , $0.185,0.210$, and $0.055 \mathrm{~V}$.

\section{SSI Interface Conditions}

SSI conditions were as follows. The temperature of cover plate, aperture- 1 , and aperture- 2 were maintained at 200,150 , and $120^{\circ} \mathrm{C}$, respectively, and the voltages of drift, focus and SSI chamber were at 70, 30, and $0 \mathrm{~V}$, respectively. Nitrogen gas was supplied from $\mathrm{N}_{2}$ gas generator AT-10NP-C (Air Tech, Yokohama, Japan) with output pressure of $0.50 \mathrm{MPa}$ and ion source inlet pressure at $0.39 \mathrm{MPa}$. Helium gas was utilized as a buffer gas with output pressure of $0.53 \mathrm{MPa}$ and ion source inlet pressure at $0.25 \mathrm{MPa}$.

\section{APCI Interface Conditions}

APCI conditions were as follows: The temperatures of the nebulizer, desolvator, aperture- 1 , and aperture- 2 were $230,380,150$, and $120^{\circ} \mathrm{C}$, respectively, and the voltages of drift, focus, needle, and detector were $70 \mathrm{~V}$, $30 \mathrm{~V}, 3.5 \mathrm{kV}$, and $500 \mathrm{~V}$, respectively. Helium was utilized as a buffer gas with output pressure of 0.53 $\mathrm{MPa}$ and with ion source inlet pressure at $0.25 \mathrm{MPa}$.

\section{HPLC Conditions}

The HPLC column used was an Shodex MSpak GF-310 4B, $50 \times 4.6 \mathrm{~mm}$ i.d. (Showa Denko, Tokyo, Japan). This column is made of a new polymer, which enables direct injection of crude biological samples, followed by size-exclusion chromatographic separation associated with slight action of partition and adsorption. The mobile phase, consisting of distilled water containing $0.09 \%$ formic acid and $20 \mathrm{mM}$ ammonium acetate (solvent $\mathrm{A}$ ), was set at a flow rate of $300 \mu \mathrm{L} / \mathrm{min}$ for $5 \mathrm{~min}$, and then gradient elution was performed using $100 \%$ A to $20 \%$ A ( $80 \%$ solvent B: Acetonitrile) over $20 \mathrm{~min}$. The mobile phase was discarded up to $5 \mathrm{~min}$, and then introduced into the mass spectrometer by a switching valve.

Table 2. Detection limits of HPLC-MS and HPLC-MS-MS with different interfaces (SSI and APCI) obtained by injection of the standard solution of haloperidol, reduced haloperidol and CPHP through the HPLC column ${ }^{\mathrm{a}}$

\begin{tabular}{|c|c|c|c|c|}
\hline & \multicolumn{2}{|c|}{ HPLC-MS } & \multicolumn{2}{|c|}{ HPLC-MS-MS } \\
\hline & $\begin{array}{l}\text { Detection limit } \\
\text { on column } \\
\text { (pg) }\end{array}$ & $\begin{array}{l}\text { Monitored } \\
\text { ion }(\mathrm{m} / \mathrm{z})\end{array}$ & $\begin{array}{l}\text { Detection limit } \\
\text { on column } \\
(\mathrm{pg})\end{array}$ & $\begin{array}{l}\text { Monitored } \\
\text { ion }^{\text {b }}(\mathrm{m} / \mathrm{z})\end{array}$ \\
\hline \multicolumn{5}{|l|}{ SSI } \\
\hline Haloperidol & 12.5 & 376 & 2.5 & 165 \\
\hline Reduced haloperidol & 25 & 378 & 12.5 & 360 \\
\hline CPHP & 1000 & 212 & 125 & 194 \\
\hline \multicolumn{5}{|l|}{$\mathrm{APCl}$} \\
\hline Haloperidol & 1000 & 376 & 250 & 165 \\
\hline Reduced haloperidol & 1000 & 378 & 375 & 360 \\
\hline CPHP & Not detectable & 212 & - & - \\
\hline
\end{tabular}

${ }^{a}$ HPLC-MS(-MS) conditions were identical to those for biological samples in this study. Detection limits were defined as the concentration at which the analyte produced a chromatographic peak with a signal-to-noise ratio of 3 . The injection onto the HPLC column was made at 18 concentrations over range of $0.25 \mathrm{pg}-62.5 \mu \mathrm{g}$ for all compounds.

bEach compound was monitored by the base peak of product ions generated by CID from each protonated molecular ion. 

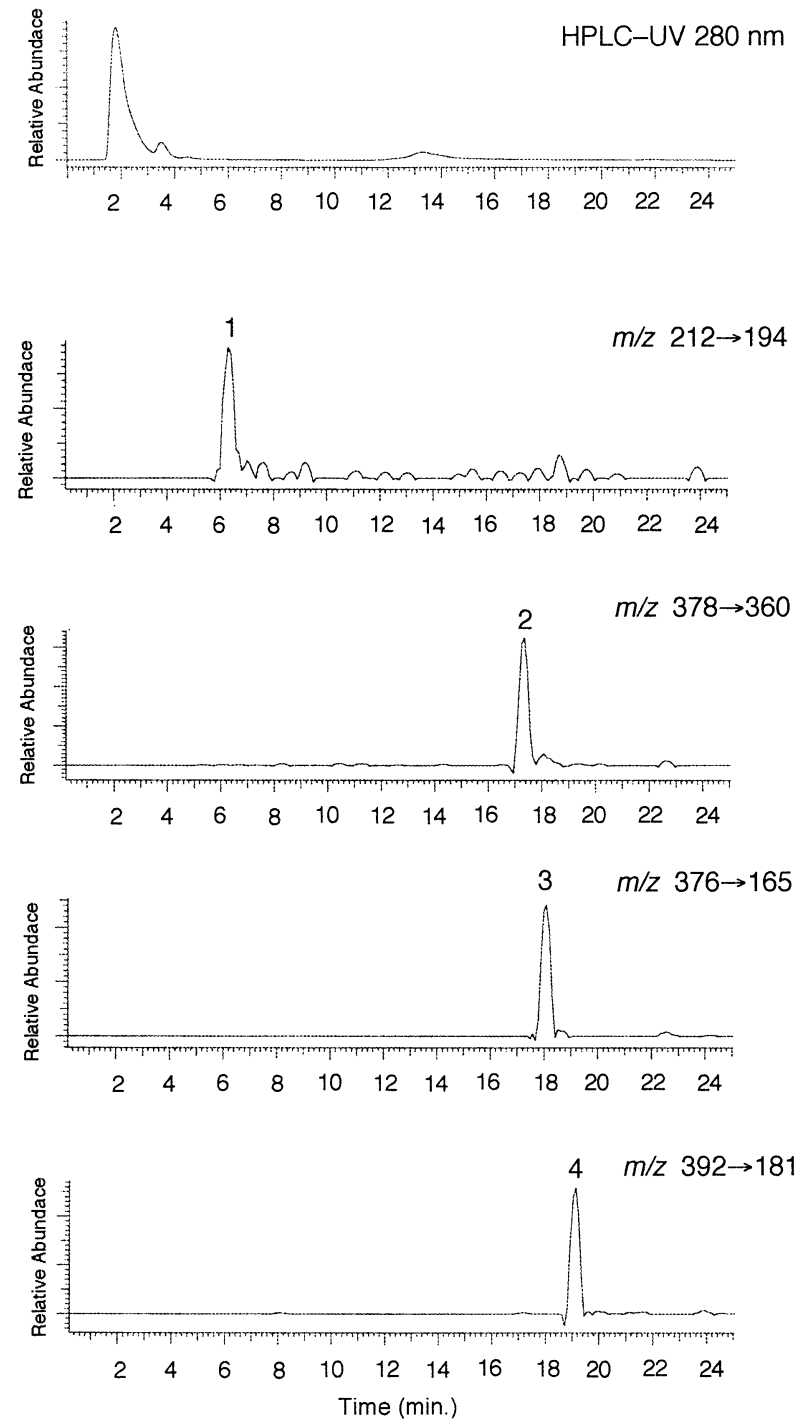

Figure 2. Typical chromatogram of HPLC-UV and mass chromatograms by HPLC-SSI-MS-MS from a human plasma sample. The amount of haloperidol, reduced haloperidol and CPHP spiked to $1 \mathrm{ml}$ plasma was $200 \mathrm{ng}$, and that of haloperidol chlorinated analog (I.S.) was $300 \mathrm{ng}$. Each compound was monitored by the base peak of product ions generated by CID from each protonated molecular ion. Sample preparation before injection onto the HPLC column is described in the text. The peak numbers: Peak 1, CPHP; Peak 2, reduced haloperidol; Peak 3, haloperidol; Peak 4, I.S.

\section{Sample Preparation of Human Plasma and Urine}

To $1 \mathrm{~mL}$ human plasma or urine in the presence of haloperidol, its two metabolites and I.S. (300 ng), $3 \mathrm{~mL}$ of the above initial HPLC mobile phase (solvent A) was added. The mixture was frozen at $-20{ }^{\circ} \mathrm{C}$. After thawing, the mixture was centrifuged at $1630 \mathrm{~g}$ for $15 \mathrm{~min}$. A $20 \mu \mathrm{L}$ aliquot of the supernatant fraction was directly injected onto the HPLC column.

\section{Results and Discussion}

Figure 1 shows mass spectra of standard haloperidol (Figure 1a), reduced haloperidol (Figure 1b), haloperi- dol chlorinated analog (Figure 1c), and CPHP (Figure 1d) obtained by SSI-MS (A) and APCI-MS (B). Mass spectra obtained with the SSI interface were similar to those with the APCI interface except for CPHP, but there seemed to be a trend of much smaller dehydration fragment ions appearing in the mass spectra with the SSI interface. In mass spectra of haloperidol for both SSI and APCI, the protonated molecular ions at $\mathrm{m} / \mathrm{z} 376$ and 378 , with an isotope ratio of $\sim 3: 1$, due to the presence of one chlorine atom, while the $\mathrm{m} / \mathrm{z} 358$ and 360 ions are derived from a dehydration process. Other major ion at $\mathrm{m} / \mathrm{z} 165$ for haloperidol is attributable to $\left[\mathrm{F}-\mathrm{C}_{6} \mathrm{H}_{4}-\mathrm{CO}-\right.$ $\left.\mathrm{C}_{3} \mathrm{H}_{6}\right]^{+}$, which results from the charge-initiated cleavage of the alkyl carbon-nitrogen bond, with expulsion of nitrogen-containing moiety as a neutral species. The protonated molecular ions and fragment peaks due to the above dehydration appeared for all compounds. The ion at $\mathrm{m} / \mathrm{z} 181$ observed for haloperidol chlorinated analog is also attributable to $\left[\mathrm{Cl}-\mathrm{C}_{6} \mathrm{H}_{4}-\mathrm{CO}-\mathrm{C}_{3} \mathrm{H}_{6}\right]^{+}$. Although we tried to record mass spectrum of CPHP with the APCI interface, no recognizable peaks could be obtained.

The typical product ions (\% intensity) obtained by CID from each protonated molecular ion for haloperidol, reduced haloperidol, haloperidol chlorinated ana$\log$, and CPHP are listed in Table 1. Each spectrum was characteristic for each parent compound and thus provides the identification tool for each compound.

Table 2 shows comparison between detection limits by HPLC-MS and HPLC-MS-MS with the different interfaces (SSI and APCI), obtained by injecting various amounts of standard compounds dissolved in the initial mobile phase under the above mentioned HPLC conditions. It was obvious that HPLC-MS-MS gave higher sensitivity than HPLC-MS for both SSI and APCI interfaces. The sensitivities by HPLC-SSI-MS-MS for haloperidol and reduced haloperidol were 100 and 30 times higher, respectively, than those by HPLC-APCI-MS-MS under our conditions. Thus, we chose the SSI interface for HPLC-MS-MS analyses of the three compounds.

Figure 2 shows chromatogram obtained by HPLC-UV (at $280 \mathrm{~nm}$ ) for monitoring proteins and nucleic acids and mass chromatograms by HPLC-SSIMS-MS obtained from human plasma. As shown in the HPLC-UV chromatogram, most of the proteins and nucleic acids in plasma were eluted within $5 \mathrm{~min}$. Haloperidol, reduced haloperidol, CPHP, and I.S. were well separated and could be determined simultaneously.

Detection limits and linearity of the methods for haloperidol and its metabolites in plasma and urine samples were carefully investigated over a concentration range of $1-800 \mathrm{ng} / \mathrm{mL}$ (14 points of concentrations). The limits of detection (single-to-noise ratio $=3$ ) for haloperidol, reduced haloperidol, and CPHP were $2.5,5$ and $75 \mathrm{ng} / \mathrm{mL}$, respectively, for both plasma and urine. Haloperidol, reduced haloperidol, and CPHP showed good linearity in the range of 5-800, 10-800, and $100-800 \mathrm{ng} / \mathrm{mL}$, respectively, for both plasma and 
urine. The correlation coefficients for these plots were found to be 0.992, 0.987, and 0.934, respectively, for plasma, and 0.992, 0.992, and 0.960, respectively, for urine.

SSI is a soft ionization method which does not require heat or high voltages to form ions; therefore, it is well suited for ionization of substances, which is sensitive to heat decomposition [1, 2]. SSI is quite different in principle from APCI and electrospray ionization (ESI); the latter is also widely used as an interface of HPLC-MS(-MS) at the present time. We thus tried analysis of CPHP by using a Finnigan MAT LCQ ion trap mass spectrometer (San Jose, CA) with the ESI interface, but no spectrum with recognizable peaks of CPHP could be obtained by mean of sequential flow injection as with the APCI interface in the present study (unpublished observation). It seems correct that SSI shows broader spectrum for organic compounds to be ionized.

Haloperidol is one of the most important neuroleptic drugs widely used. The drug and/or its metabolites were analyzed by gas chromatography (GC)-MS(-MS) [4, 5], HPLC-APCI-MS [6], HPLC-ESI-MS(-MS) [6-10], and HPLC-thermospray-MS-MS [11]. A hydrophobic drug is usually metabolized in the body to hydrophilic metabolite(s), and excreted into urine. It is therefore important to analyze a hydrophobic drug and its hydrophilic metabolite(s) simultaneously by a single interface of HPLC-MS(-MS). However, CPHP (a hydrophilic metabolite) could not be analyzed with the popular interfaces such as APCI and ESI. In this respect, our HPLC-SSI-MS-MS method seems recommendable.

\section{Conclusions}

To our knowledge, this is the first trial to compare the SSI with the APCI as an interface of HPLC-MS(-MS) for analysis of a drug and its metabolite(s). The sensitivities by HPLC-SSI-MS-MS for haloperidol and reduced haloperidol were 100 and 30 times higher, respectively, than those by HPLC-APCI-MS-MS under our conditions; CPHP could be detected by HPLC-SSI-MS(-MS), but not by HPLC-APCI-MS(-MS). Based on the comparison, we have established a detailed procedure for simultaneous analysis of haloperidol and its two metabolites in human plasma and urine by the HPLC-SSIMS-MS coupled with a special HPLC column.

\section{Acknowledgments}

The authors wish to express their deep gratitude to other members of the Department of Aichi Medical University School of Medicine for their skillful cooperation.

\section{References}

1. Hirabayashi, A.; Sakairi, M.; Koizumi, H. Anal. Chem. 1994, 66, 4557-4559.

2. Hirabayashi, A.; Sakairi, M.; Koizumi, H. Anal. Chem. 1995, 67, $2878-2882$.

3. Hirabayashi, Y.; Hirabayashi, A.; Takada, Y.; Sakairi, M.; Koizumi, H. Anal. Chem. 1998, 70, 1882-1884.

4. Fang, J.; Gorrod, J. W.; Kajbaf, M.; Lamb, J. H.; Naylor, S. Int. J. Mass Spectrom. Ion Process. 1992, 122, 121-131.

5. Hattori, H.; Suzuki, O.; Brandenberger, H. J. Chromatogr. 1986, 382, 135-145.

6. Igarashi, K.; Sigee, Y.; Kasuya, F.; Fukui, M. Jpn. J. Forensic Toxicol. 1997, 15, 44-54.

7. Seno, H.; Hattori, H.; Ishii, A.; Kumazawa, T.; Suzuki, K. W.; Suzuki, O. J. Chromatogr. B 2000, 746, 3-9.

8. Weinmann, W.; Wiedemann, A.; Eppinger, B.; Renz, M.; Svoboda, M. J. Am. Soc. Mass Spectrom. 1999, 10, 1028-1037.

9. Hempenius, J.; Steenvoorden, R. J. J. M.; Lagerwerf, F. M.; Wieling, J.; Jonkman, J. H. G. J. Pharm. Biomed. Anal. 1999, 20, 889-898.

10. Hoja, H.; Marquet, P.; Verneuil, B.; Lotfi, H.; Dupuy, J. L.; Pénicaut, B.; Lachâre, G. J. Chromatogr. B 1997, 688, 275-280.

11. Verweij, A. M. A.; Hordijk, M. L.; Lipman, P. J. L. J. Chromatogr. B 1996, 686, 27-34. 